\title{
Supratentorial Multiple Sclerosis Lesions Affect the Blink Reflex Test
}

\author{
Efthimios H. Mikropoulos*,1, Afroditi A. Papathanasiou ${ }^{2}$, Georgios Hadjigeorgiou ${ }^{1}$, \\ Evangelia Tsironi ${ }^{3}$ and Alex Papadimitriou ${ }^{1}$
}

\author{
${ }^{I}$ Department of Neurology, Faculty of Medicine, School of Health Sciences, University of Thessaly, P.O. Box 1400, \\ Mezourlo Hill, Larissa, Greece \\ ${ }^{2}$ Department of Biomathematics, Faculty of Medicine, School of Health Sciences, University of Thessaly, P.O. Box 1400, \\ Mezourlo Hill, Larissa, Greece \\ ${ }^{3}$ Department of Ophthalmology, Faculty of Medicine, School of Health Sciences, University of Thessaly, P.O. Box 1400, \\ Mezourlo Hill, Larissa, Greece
}

\begin{abstract}
Introduction: The Blink Reflex Test (BRT) is a neurophysiological examination used for evaluation of brainstem reflex circuits. MRI is the most precise modality for evaluation of MS lesion anatomy. Our study objective was to investigate how the functional results of the neurophysiological BRT relate to the anatomy of MS lesions in routine MRI studies.

Methods: 65 MS patients underwent the BRT within 2 months of a brain MRI showing demyelinating lesions.

Results: The overall sensitivity of the BRT was $90.8 \%$, while in patients with at least one brainstem lesion and no brainstem lesions it was $91.4 \%$ and $90 \%$, respectively.

Discussion: The presence of brainstem lesions does not significantly affect BRT sensitivity. This points to the influence of supratentorial MS lesions on the BRT. Gender, age, disease duration, type of MS, acuteness of an MS event and whether MS diagnosis was recent or not were not variables affecting the results.
\end{abstract}

Keywords: Blink Reflex Test, Multiple Sclerosis, MRI Brain, Supratentorial Lesions, Brainstem.

\section{INTRODUCTION}

The Blink Reflex Test (BRT) [1-3] is a neurophysiological examination in which unilateral electrical stimulation of the nerve trunks of the supraorbital branches of the trigeminal nerve in the forehead produces the afferent volley of a reflex, the efferent loop of which leads via the facial nerves to bilateral contraction of the orbicularis oculi muscles. Though it has some similarity to the clinical corneal reflex in which stimulation is applied to the nerve endings of the trigeminal nerve in the cornea, differences have been described $[4,5]$. The BRT normally produces two responses: an early (8-13 ms) unilateral R1 and a late (23-44 ms) bilateral R2. The $\mathrm{R} 1$ response is synchronous and does not habituate [5-8] since it is oligosynaptic [9]. It involves the main sensory nucleus of $\mathrm{V}[4,5,10,11]$ in the lateral midpontine and lower pontine regions and the ipsilateral facial nucleus (more specifically, the intermediate subnucleus [5,12-14]). The R2 response is asynchronous because its central loop consists of a polysynaptic chain $[4,5,15,16]$ with fibers 1$)$ descending in the nucleus of the spinal tract of $\mathrm{V}$ from the pons to the caudal medulla before ascending ipsilaterally to reach the facial nucleus and produce the ipsilateral R2 response and also 2)

\footnotetext{
*Address correspondence to this author at 41, Ypsilantou Str., Athens 10676, Greece; Tel: (30)-697-588-3151; Fax: (30)-210-674-6756; Email: timikro@pol.net
}

crossing at the caudal medullary level to ascend contralaterally within the lateral propriobulbar system of the reticular formation, reaching the contralateral facial nucleus and producing the contralateral $\mathrm{R} 2$ response.

Prior to introduction of MRI studies in neurology, the BRT was one of the diagnostic methods used in conjunction with clinical and other ancillary tests to diagnose MS. As the superior sensitivity of MRI studies was established, the use of the BRT was reduced in clinical practice, as were all other electrodiagnostic tests. In this study, we set out to investigate how the functional results of the BRT relate to the anatomy of MS lesions imaged in routine MRI studies.

\section{MATERIALS AND METHODOLOGY}

\section{Participants}

The patients examined in this prospective cohort were derived from our Neurology Clinic. One of the authors selected them based on their meeting the McDonald criteria [17] for diagnosis of definite MS. The patients selected were out of a random pool of MS patients showing up in the Neurology Clinic in the period allocated to testing (regular check-ups, relapses, de novo cases). Informed consent was requested and provided by all of them. The author performing the BRT was blinded as to the brain MRI results of the patients examined until after the collection of all data. We examined individuals who had just been diagnosed with MS, as well as patients who already carried the diagnosis. Purely spinal forms of MS with no brain lesions were excluded. The 
clinical presence of a normal or abnormal corneal reflex was not considered since it is not exactly the same as the blink reflex as far as the type of receptors and reflex circuit involved are concerned [4,5]. Furthermore, the symptoms and clinical findings of the patients, whether suggesting brainstem involvement or not, were not taken into consideration for reasons explained in a later section. Sixty-five patients were examined (44 women and $21 \mathrm{men}$ ). Their ages varied from $17-71$ years. Of these $65,38 / 65(58.5 \%)$ were under 40 , while $27 / 65(41.5 \%)$ were aged over 40 . Based on the detailed history of disease onset of all patients, their disease duration was estimated and found to be under 3 years in $36 / 65(55.4 \%)$ and over 3 years in 29/65 (44.6\%). Based on their clinical type of MS, 45/65 (69.2\%) patients had Relapsing-Remitting MS (RRMS), 13/65 (20\%) had Secondary Progressive MS (SPMS) and 7/65 (10.8\%) had ProgressiveRelapsing MS (PRMS). In 40/65 (61.5\%) the BRT was performed within 1 week of the onset of diagnosis of MS [13/65 (20\%) patients] or of a clinical MS relapse [22/65 (33.8\%) RRMS patients and 5/65 (7.7\%) PRMS] and before steroids or any other treatment was given, while in $25 / 65(38.5 \%)$ the timing of performance of the BRT was not related to a recent clinical MS event. Definite MS was just diagnosed (and in retrospect confirmed) in 13/65 (20\%) of patients examined (no treatment had been initiated at the time of performance of the BRT), while in 52/65 (80\%) the diagnosis had already been established.

\section{Radiological Criteria and Patient Groups}

MRI exams used for the study were performed a) as part of the diagnosis in de novo MS patients (13/65), b) within 1 week of clinical relapse and BRT performance in 22/65 patients with established RRMS and in 5/65 patients with PRMS and c) within 2 months of the BRT in the 13/65 patients with SPMS, 10/65 patients with RRMS and 2/65 patients with PRMS who had no recent clinical MS event. This time frame of 2 months was chosen based on knowledge of temporal evolution of gadolinium enhancement of lesions on MRI. The results of MRI exams were not known to the person performing the BRT at the time of testing. The criteria of the International Consortium of MS centers [18] were used. MR imaging was performed on a Phillips Intera 1.5 T scanner with a standard head coil and included the following pulse sequences: 1) 3 plane scout set up axial sections through the subcallosal line, 2) Sagittal fast FLAIR, 3) Axial FSE PD/T2 (TE 30/80), 4) Axial Fast FLAIR, 5) Axial pregadolinium T1 weighted images, 6) Axial post gadolinium T1 weighted images obtained 5 minutes after the injection of $0.1 \mathrm{mmole} / \mathrm{kg}$ of gadolinium based contrast agent. The axial slices were obtained with a section thickness of $3 \mathrm{~mm}$ with no intersection gap when feasible. The findings of each MRI exam were analyzed and reviewed in order to determine: 1) whether there was at least one brainstem lesion, 2) if there was a lesion in the brainstem, whether it showed gadolinium enhancement, 3) if there was a lesion in the brainstem, whether it was in the medulla, the pons or the midbrain, 4) whether any of the other lesions in the cerebral hemispheres showed gadolinium enhancement. Based on their brain MRI findings, the 65 MS patients examined were classified in the following categories: 1) 22 patients (33.9\%) with no brainstem lesions and no gadolinium enhancement in any of the hemispheric lesions, 2) 8 patients $(12.3 \%)$ with no brainstem lesions, but with gadolinium enhancement in at least one hemispheric lesion, 3) 21 patients (32.3\%) with nonenhancing brainstem lesions, whose other hemispheric lesions were also non-enhancing, 4) 9 patients (13.9\%) with non-enhancing brainstem lesions and at least one enhancing hemispheric lesion, 5) 3 patients $(4.6 \%)$ with enhancing lesions in the brainstem and the hemispheres, 6) 2 patients (3\%) with enhancing lesions in the brainstem, but not in the hemispheres.

\section{Blink Reflex Test Setup and Protocol}

The BRT was performed according to the standard methods suggested by the International Federation of Clinical Neurophysiology [19]. The Nihon-Koden, Neuropack- $\Sigma$ model was used for testing. All tests were performed by the same person. The patients were in supine position, in a relaxed state and with eyes open. A ground electrode was placed on the patients' forehead or chin. The active recording electrode was placed on the inferior orbital part of the orbicularis oculi, just lateral to the vertical line that may be drawn with the pupils in midposition. The reference recording electrode was placed lateral to the lateral canthus of each eye. Recording electrodes were placed on both sides. The machine settings were as follows: sweep speed 10 $\mathrm{ms} /$ division, sensitivity $100-200 \mu \mathrm{V} /$ division, motor filter settings $10 \mathrm{~Hz}$ and $10 \mathrm{kHz}$. Facial nerve studies were initially performed for each patient to ensure that normal motor latency and amplitude could be obtained on each side and that there was no damage to the peripheral part of the efferent loop of the reflex. All patients examined had normal facial nerve studies. The supraorbital branches of the trigeminal nerve were stimulated on each side with a stimulus of 5$25 \mathrm{~mA}$ intensity, $0.1 \mathrm{~ms}$ duration and no faster than $1 / 30 \mathrm{~s}$ frequency. The stimulus intensity was increased by $5 \mathrm{~mA}$ until a reliable response was obtained. Bilateral recordings were made simultaneously. In order to obtain both the shortest R1 and R2 latency and an unambiguous response once each patient's threshold stimulus intensity was obtained, 410 responses were superimposed from each side and one was chosen (see discussion).

\section{Blink Reflex Test Interpretation}

Twenty three normal subjects were examined ( 8 males, 15 females, age 19-42) after informed consent was obtained. Abnormal latency values were established as follows: R1 $>13 \mathrm{~ms}$, ipsilateral R2 $>41 \mathrm{~ms}$, contralateral R2 $>44 \mathrm{~ms}$. The values obtained are in accordance with published literature $[1,2,5,13]$. We considered responses to be abnormal when 1) they could not be elicited or 2) were delayed beyond the normal values. As previously described elsewhere $[1,5,11,13]$, additional criteria utilized were: 3 ) unilateral delay of R1 $>1.2$ ms compared to other side, 4) side differences of R2 $>5 \mathrm{~ms}$ between the ipsilateral and contralateral responses, obtained with unilateral stimulation, 5) side differences of R2 $>7 \mathrm{~ms}$ on a given side, obtained with right and left sided stimulation.

\section{Statistical Analysis}

The sensitivity of the test in all categories and the respective $95 \%$ confidence interval (CI) was calculated. The association between BRT outcome and categorical variables was tested using the chi-square test. 
Table 1. Blink Reflex Test Results

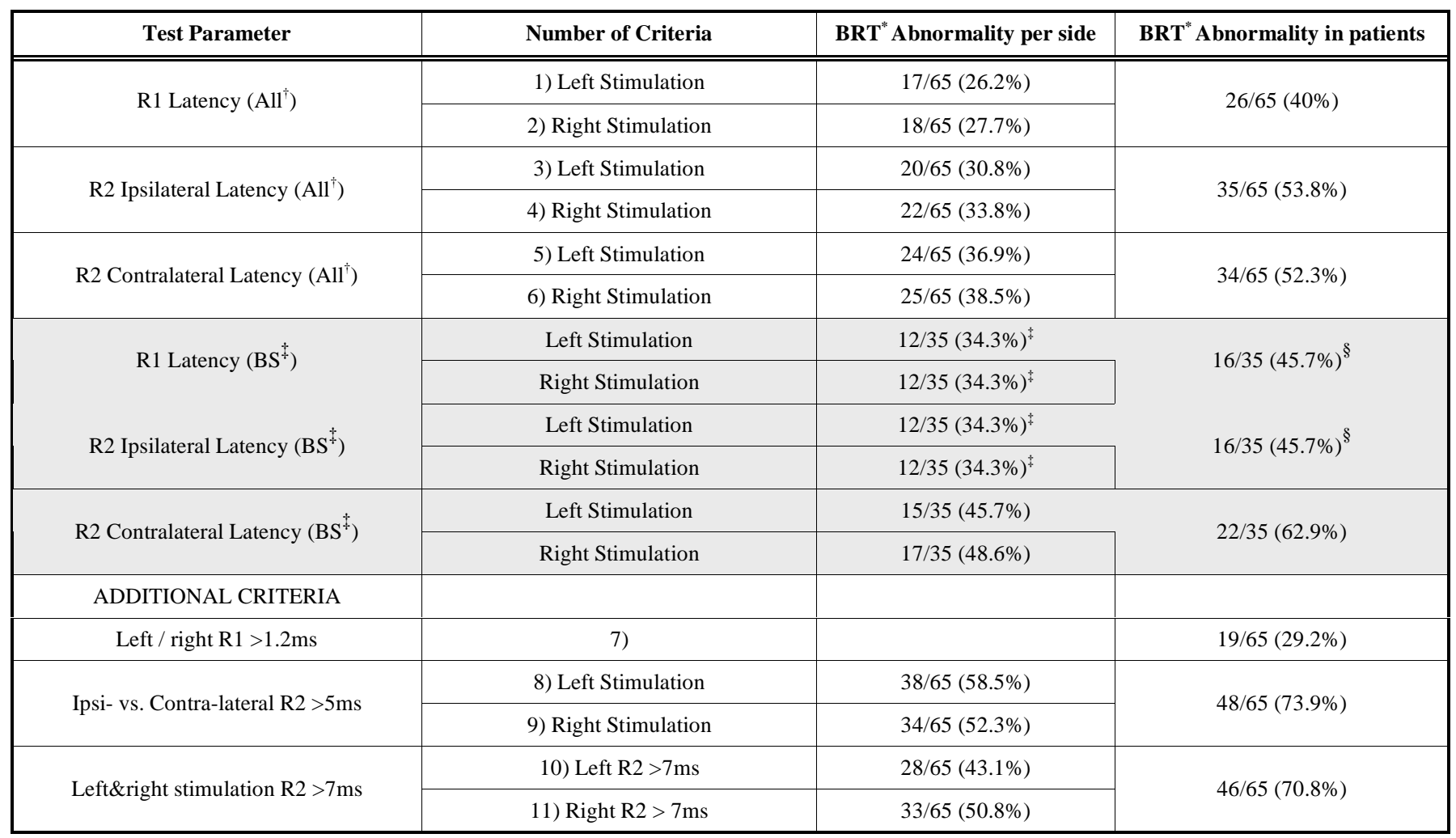

"BRT: Blink Reflex Test.

${ }^{\dagger}$ All: All patients examined, regardless of brainstem involvement or not.

*BS: Brainstem involvement, regardless of concurrent hemispheric involvement.

${ }^{\S}$ Note: it is a pure numerical coincidence that that percentage of abnormalities of R1 and ipsilateral R2 latencies in the brainstem are the same, as the patients from whom they were derived were not.

\section{RESULTS}

\section{Test Results}

Table 1, in its upper section, shows the BRT results of all patients. The R1 latency was abnormal in 26/65 (40\%) patients. The ipsilateral R2 latency was abnormal in $35 / 65$ $(53.8 \%)$. The contralateral R2 latency was abnormal in 34/65 $(52.3 \%)$ patients. The reason that the number of patients showing abnormalities of each parameter is not the sum of abnormalities found on each side is because the same patient often showed abnormalities on both sides.

Table 1, in its lower section, also shows the sensitivity of the additional criteria, obtained by calculations based on the BRT results recorded. It is worth mentioning that the additional criteria employed that are based on mathematical calculations constituted the sole basis for considering the BRT as positive in only $4 / 65(6.2 \%)$ patients.

The number of criteria used to define the BRT as positive is shown in Table 2. Even though the above described six criteria were used, the number of criteria that arises from bilateral evaluation of results is eleven. In the vast majority of patients, several criteria were met confirming the positivity of the BRT.

\section{Overall Sensitivity}

The BRT was overall positive in $59 / 65$ of patients with MS. Thus, the sensitivity of the test was Se $=90.8 \%$ (95\% CI: $83.7-97.8)$. The test was negative in $6 / 65$ patients $[9.2 \%$ (95\% CI: 2.2-16.3)].

\section{Sensitivity in Patient Subpopulations, Based on MRI Data}

Table 3 shows the sensitivity of the BRT for the different outcomes of brain MRI. Of the 65 patients examined, 35/65 $(53.9 \%)$ had at least one lesion in the brainstem on MRI, while 30/65 (46.1\%) had no brainstem lesions. In the 35 patients with at least one brainstem lesion, the sensitivity of the BRT was $\mathrm{Se}=91.4 \%$ (95\% CI: 82.2-100), while $8.6 \%(95 \%$

Table 2. Number of Criteria Fulfilled for Considering the Blink Reflex Text as Abnormal

\begin{tabular}{|c|c|c|c|c|c|c|c|c|c|c|c|}
\hline $\begin{array}{c}\text { Number of criteria fulfilled for } \\
\text { abnormal BRT }\end{array}$ & 1 & 2 & 3 & 4 & 5 & 6 & 7 & 8 & 9 & 10 & 11 \\
\hline Patient number & 8 & 10 & 6 & 5 & 4 & 10 & 4 & 7 & 2 & 2 & 1 \\
\hline Patient percentage ${ }^{\dagger}$ & $13.6 \%$ & $16.9 \%$ & $10.2 \%$ & $8.5 \%$ & $6.8 \%$ & $16.9 \%$ & $6.8 \%$ & $11.9 \%$ & $3.4 \%$ & $3.4 \%$ & $1.7 \%$ \\
\hline
\end{tabular}

"BRT: Blink Reflex Test.

${ }^{\dagger}$ Patient percentage: Patient number / 59 (59 patients had an abnormal BRT). 
Table 3. Sensitivity of the Blink Reflex Test for the Different Outcomes of Brain MRI

\begin{tabular}{|c|c|c|}
\hline \multicolumn{2}{|c|}{ MRI" brain (gold standard) } & No. of Positive/ Total \\
\hline \multicolumn{2}{|c|}{ Overall } & $59 / 65$ \\
\hline \multirow{2}{*}{ Presence of $\mathrm{BS}^{\S}$ lesions } & none & $\begin{array}{c}27 / 30 \\
90.0 \%(79.3-100 \%)\end{array}$ \\
\hline & $\geq 1$ & $\begin{array}{c}32 / 35 \\
91.4 \%(82.2-100 \%)\end{array}$ \\
\hline \multirow{2}{*}{ Gadolinium enhancement, overall } & none & $\begin{array}{c}40 / 43 \\
93 \%(85.4-100 \%)\end{array}$ \\
\hline & in $\geq 1$ lesions & $\begin{array}{c}19 / 22 \\
86.4 \%(72-100 \%)\end{array}$ \\
\hline \multirow{2}{*}{$\begin{array}{l}\text { Gadolinium enhancement, } \\
\text { specifically }\end{array}$} & $\begin{array}{l}\text { in } \mathrm{BS}^{\S} \text { lesions } \\
\text { (and hemispheric) }\end{array}$ & $\begin{array}{c}5 / 5 \\
100 \%\end{array}$ \\
\hline & only in hemispheric lesions & $\begin{array}{c}14 / 17 \\
82.4 \%(64.2-100 \%)\end{array}$ \\
\hline
\end{tabular}

*MRI: Magnetic Resonance Imaging.

${ }^{\dagger}$ BRT: Blink Reflex Test.

${ }^{\ddagger} \mathrm{CI}$ : Confidence Interval.

${ }^{\S}$ BS: Brainstem.

CI: 0-17.8) had a negative test. In the 30 patients with no brainstem lesions, the sensitivity of the BRT was $\mathrm{Se}=90 \%$ (95\% CI: 79.3-100), while 10\% (95\% CI: 0-20.7\%) had a negative test.

The number of patients studied in whom there was no gadolinium enhancement in any of the imaged lesions was 43 and, in these patients, the sensitivity of the BRT was Se=93\% (95\% CI: 85.4-100). Twenty two patients had at least one gadolinium enhancing lesion anywhere in the brain, whether in the hemispheres or the brainstem, and the BRT sensitivity was $\mathrm{Se}=86.4 \%$ (95\% CI: 72-100).

In 5 of the MS patients studied, there was gadolinium enhancement in at least one brainstem lesion, regardless of the presence or absence of gadolinium enhancement in other lesions in the cerebral hemispheres. In all these patients $(100 \%)$, the BRT was positive.

The number of patients studied in whom there was gadolinium enhancement only in cerebral hemispheric but not in brainstem lesions was 17, regardless of the presence of a non-enhancing lesion in the brainstem. The BRT sensitivity was $\mathrm{Se}=82.4 \%$ (95\% CI: 64.2-100).

\section{Effect of Site of Brainstem Lesion}

As mentioned above, brainstem lesions were present in 35 of the MS patients examined. The majority had only one lesion, while some had more than one. Most involved in our random series of MS patients was the pons and 19/21 ( $\mathrm{Se}=90.5 \%$, 95\% C.I.: 78-100) patients with one or more pontine lesions had an abnormal BRT. Both the pons and the medulla were involved in 8 patients, all of whom had an abnormal BRT. The medulla alone was involved in just 2 patients, both of whom had an abnormal BRT. Of the 2 patients who had a solitary midbrain lesion, only one had an abnormal BRT. A patient with lesions in the midbrain and medulla and another patient who had a lesion in all three divisions of the brainstem, both had an abnormal BRT. Table $\mathbf{4}$ shows the distribution of lesions within the brainstem and the associated BRT results.

Table 4. Site of Brainstem Lesions and Blink Reflex Test Results

\begin{tabular}{|c|c|c|}
\hline \multirow{2}{*}{ MRI* Brain Site of Brainstem Lesions } & \multicolumn{2}{|c|}{ BRT $^{\dagger}$ Results Number of patients } \\
\cline { 2 - 3 } & Abnormal BRT & $2 / 21$ \\
\hline \hline Pons (only) & $19 / 21$ & $0 / 8$ \\
\hline Pons + Medulla & $8 / 8$ & $0 / 2$ \\
\hline Medulla (only) & $2 / 2$ & $0 / 1$ \\
\hline Medulla + Midbrain & $1 / 1$ & $1 / 2$ \\
\hline Midbrain (only) & $1 / 2$ & $0 / 1$ \\
\hline Midbrain+Pons+Medulla & $1 / 1$ & $3 / 35(8.6 \%)$ \\
\hline TOTAL & $32 / 35(91.4 \%)$ & \\
\hline
\end{tabular}

*MRI: Magnetic Resonance Imaging

${ }^{\dagger}$ BRT: Blink Reflex Test. 
Table 1, in its middle shaded region, shows the detailed BRT results in this subgroup of patients. Among the $35 \mathrm{MS}$ patients in whom brainstem lesions were found in MRI, the R1 latency was abnormal in 16/35 (45.7\%) patients. The ipsilateral R2 latency was abnormal in a different group of 16/35 (45.7\%) patients. The contralateral R2 latency was abnormal in 22/35 (62.9\%).

\section{Effect of MRI Enhancement of Brainstem Lesion}

As mentioned above, all 5 patients examined who had at least one enhancing brainstem lesion (regardless of the presence or absence of gadolinium enhancement in other lesions in the cerebral hemispheres) had an abnormal BRT. Of these patients, two had lesions only in the pons, two had lesions in the pons and the medulla and one had a lesion only in the medulla. Interestingly, the left and right R1 latencies were normal in all of these patients and subsequent revision of their MRIs showed that 3 out of 4 pontine lesions were in the upper pons (not in the mid- or lower pons, where R1 abnormalities have been localized to in the literature). The ipsilateral R2 latencies were bilaterally abnormal in just 2 of the patients and, of note, their contralateral R2 latencies were bilaterally normal (both patients had pontine lesions; one also had a medullary lesion). The contralateral R2 latencies were abnormal in all 3 patients with a medullary lesion (of whom one also had a pontine lesion) and in 1 patient with a single pontine lesion.

Of the other 30 patients with a brainstem lesion that showed no gadolinium enhancement, 27 ( $\mathrm{Se}=90 \%$, 95\% C.I.: 79.3-100) had an abnormal BRT and 3 patients $(\mathrm{Se}=10 \%$, 95\% C.I.: 0-20.7) had a normal BRT.

Effect of Gender, Age, Disease Duration, Clinical Form of MS, Acute MS Event, Recent MS Diagnosis

Table 5 shows the association results between BRT outcome and the demographic data of the patients examined. No association was found between the BRT outcome and 1) the gender $[\mathrm{p}=0.66], 2)$ age under and over $40[\mathrm{p}=0.99]$, 3) disease duration under and over 3 years $[\mathrm{p}=0.99]$, 4) clinical type of MS (RRMS, SPMS, PRMS) [p=0.63], 5) the presence of an acute MS clinical event (one week or less) before BRT testing (no treatment initiated before BRT) vs. not $[\mathrm{p}=0.99], 6) \mathrm{MS}$ being a recent diagnosis (before any treatment was provided to them) vs. being an already established diagnosis $[\mathrm{p}=0.59]$.

\section{DISCUSSION}

\section{BRT in MS}

The first conclusion was that $90.8 \%$ of our patients, all of whom had an established diagnosis of MS and brain lesions, were found to have a positive BRT. A literature review reveals a relatively small number of studies concerning the use of the BRT in MS [13,20-33]. Most of these were conducted before 1986 and the results applied to patients in whom the diagnosis had been established or presumed without the possibility of MRI correlation. The sensitivity of the BRT in these studies was 26-78\%, but direct comparison with our study cannot be made. It has been suggested that the diversity of sites of lesions within the brainstem is responsible for the diversity in responses between patients [34]. In a study of 21 patients with a diagnosis of definite MS who did not have clinical symptoms or signs referable to the brainstem, the sensitivity of the test was only $40 \%$ [32]. MRI had been performed in all of them, but the findings were not further analyzed. In a recent study published that has some similarity to ours in that MRI findings were considered, the BRT was positive in $75 \%$ of 40 patients with RRMS and $83.3 \%$ of 6 patients with SPMS [33]. However, for reasons explained below, the principal aim of our study was not to investigate a possible correlation between the results of the BRT and clinical findings, but with imaging data provided by the routine MRI protocols that are widely used today.

Table 5. Association Results Between Blink Reflex Test Outcome and Demographic Data

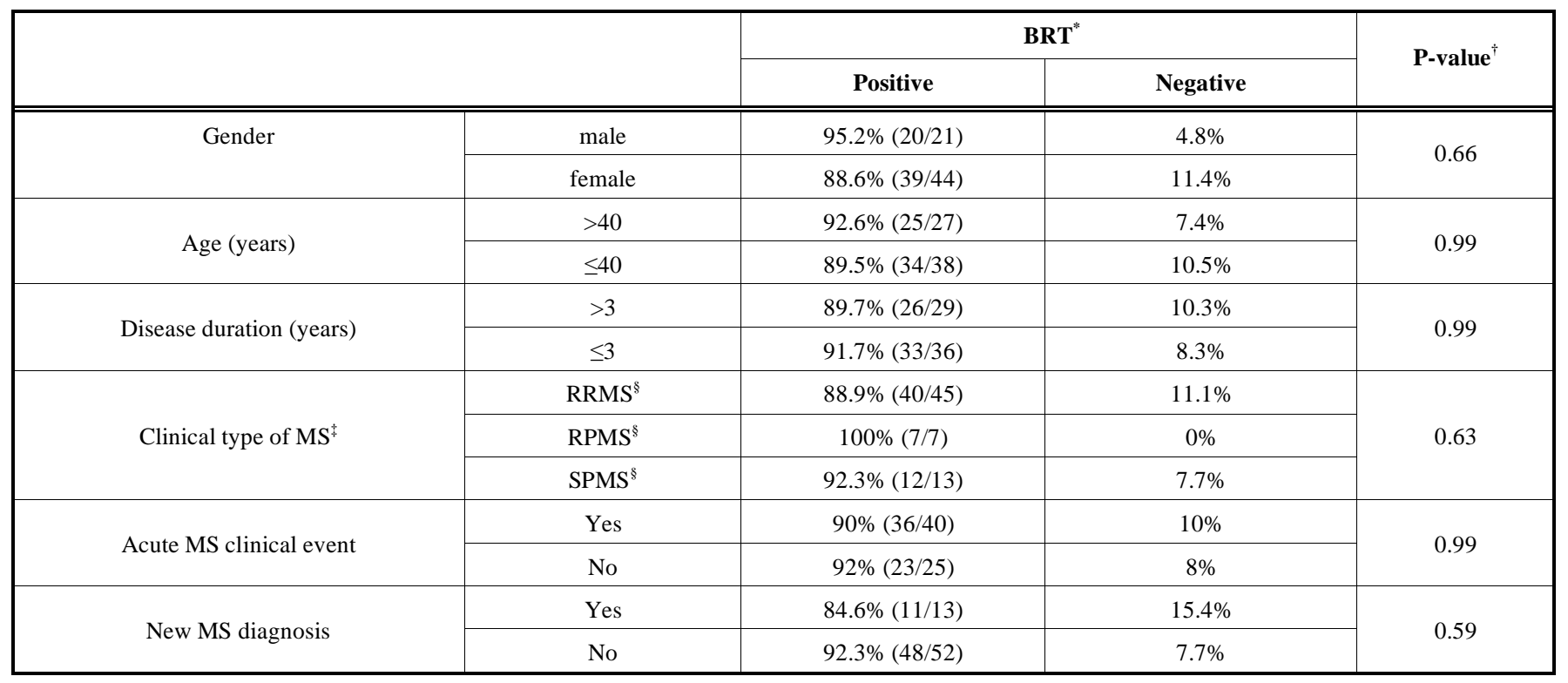

"BRT: Blink Reflex Test.

"Using the chi-squared test.

${ }^{*}$ MS: Multiple Sclerosis.

${ }^{8}$ Clinical Types of MS: RRMS, Relapsing-Remitting; RPMS, Relapsing Progressive; SPMS, Secondary Progressive. 
It was surprising to find such a high sensitivity (90.8\%) of BRT in our MS patients. In our study, the BRT was performed by the same person who was unaware of the results of MRI exams, as to the presence of brainstem lesions or gadolinium enhancement. Statistical analysis clearly showed the validity of our results through different radiological and demographic variables, each variable involving groups of different patients. In addition, several criteria were used and were fulfilled in most patients in order to consider the BRT as positive (Table 2). If we had only considered abnormal latencies of R1, ipsilateral and contralateral R2 as criteria of positivity without including criteria involving mathematical calculations, the BRT would have been found positive in $55 / 65(\mathrm{Se}=84.6 \%, 95 \%$ CI: 75.8-93.4) instead of 59/65 ( $\mathrm{Se}=90.8 \%, 95 \%$ CI: 83.7-97.8). This sensitivity would have been in the high range of that published by others, the vast majority of whom did not employ the additional criteria. Considering the mean latencies of R1 and R2 of each patient instead of choosing the most unambiguous trace with the shortest latency, as we did, may also have produced different results that would explain the diversity of findings reported in various studies. However, this data could not be retrieved when the testing was completed and the above results were obtained. It cannot be answered if this methodological difference can account for the discrepancy between our study and others, but future investigators planning to conduct similar studies should probably consider the mean latencies of R1 and R2 for each patient or even investigate whether the two different approaches significantly affect the results. Perhaps part of the explanation lies in differences between current and older machines used for electromyography. In the end, due to the study design differences and lack of more details on possible technical and interpretation differences of testing, we cannot adequately explain the discrepancy in sensitivity between our study and others. But after all, an adequate explanation for the wide variability in sensitivity reported in other existing studies (approximately 26-83.3\%) has never been provided, given that all published data are supposed to have been derived by experienced investigators trying their best to adhere to similar testing protocols.

\section{Impact of Brainstem Demyelinating Lesions}

The fact that consistency is noted between the overall sensitivity of the BRT $(90.8 \%)$ and the sensitivity of this test in patients with (91.4\%) and without (90\%) lesions in the brainstem, suggests that the mere presence of imaged lesions in the brainstem on a conventional MRI does not have a significant effect on the diagnostic value of the BRT.

A simplistic expectation in such a study would be that since the BRT is mediated mainly by the brainstem, a higher sensitivity should be revealed in patients with imaged brainstem pathology. However, an explanation for our results may lie in the importance of the influence of supratentorial lesions of the midbrain and hemispheres on the BRT, with both R1 and, especially, R2 waves known to be affected in patients with vascular lesions, mostly acute and isolated. Such influences on R1 $[5,13,35,36]$ are considered to be more limited and acute than those of R2. Therefore, in patients with vascular lesions, an isolated abnormal R1 response is generally thought to strongly correlate to ipsilateral lateral midpontine or lower pontine lesions [1,4,5,10-13]. The same does not apply with the R2 responses in which supratentorial influences of lesions in the midbrain and hemispheres, especially the lower postcentral region, affect the results to a degree that does not allow R2 abnormalities to reliably be attributed to bulbar lesions $[1,5,15,16,35,37$ 42]. This may explain why R2 abnormalities were more frequently encountered in our study than R1 abnormalities, whether examining the results of all patients or just the results of patients with imaged brainstem pathology (Table 2). The finding that the percentage of R1 and R2 abnormalities was higher in the group of patients with imaged brainstem pathology compared to all patients in the study may still be in accordance with the fact that the main anatomic circuitry involved in BRT is in the brainstem. The above results may finally suggest that MRI may reflect brainstem involvement more appropriately than BRT in MS. Based on our findings and on the presumption that in MS the pathology is not as limited and the clinical symptomatology is not necessarily as well-defined as that of a patient with an acute brainstem infarction (especially one who is more qualified to be studied with BRT localization studies based on the absence of multiinfarct disease), we do not think that extracting results about the localizing value of R1 and R2 from patients with MS and acute brainstem lesions is similar. Furthermore, even though studies of patients with brainstem infarctions have shown an abnormal R1 response to have a strong localizing value pointing to ipsilateral midpontine to lower pontine lesions, a correlative MRI study in patients with brainstem infarctions failed to confirm this [43]. The above data suggest that research studies of the blink reflex involving heterogeneous groups of diseases should possibly be avoided.

Another explanation for our findings may lie in the MRI techniques used today for imaging MS lesions. In our study, standard MRI techniques were accepted for the imaging of lesions in the brainstem and hemispheres. It is possible that as some newer imaging techniques such as diffusion tensor imaging (fractional anisotropy, tractography), magnetization transfer ratio (MTR) imaging, dual inversion recovery imaging, MR spectroscopy, functional MRI and others may be more widely employed and increase the sensitivity of MRIs of the brain, there will be better correlation of imaging data with neurophysiological or clinical findings.

One may wonder about the correlation of clinical brainstem signs to BRT results. After considerable thought, the symptoms and clinical findings, whether suggesting brainstem involvement or not, were not taken into consideration. The reason for this choice is not only that symptoms and clinical findings attributed solely to brainstem involvement constitute a minority, especially at onset, but more importantly that the majority of ophthalmokinetic, motor and sensory symptoms - especially as time from diagnosis goes by may also be caused by lesions anywhere along the complex tracts [44] that carry or influence these modalities supratentorially. In our opinion, this often makes the distinction of which of many lesions produces these symptoms in MS ambiguous and often too subjective for statistical analysis. The fact that studies in patients with MS have shown a frequent association of internuclear ophthalmoplegia with R1 abnormalities (that is still limited to $1 / 3$ of these patients) [10,11] does not mean that one can draw conclusions about more subtle or rare ophthalmokinetic abnormalities and their anatomic correlates, especially given the usual dissemination of lesions in MS. 


\section{Impact of Gadolinium Enhancement on MRI}

As shown in Table $\mathbf{3}$, even though one might expect a higher BRT sensitivity in patients whose MRI showed gadolinium enhancement as an indication of more active disease, this was not found to be true. It is interesting, however, that all five patients with at least one gadolinium enhancing lesion in the brainstem had a positive BRT. This result does not have the desired statistical significance due to the small number of patients, but represents what a clinician would inherently expect by correlating neurophysiology with neuroanatomy.

\section{Impact of Patient Demographic and Clinical Parameters}

The BRT results were not affected by the gender of the patients, whether their age was under or over 40, whether the diagnosis of MS was recent or not, whether an acute MS event had occurred, whether disease duration was less or more than 3 years, and whether they were currently classified in one or other type of MS. These findings are interesting considering that younger patients are generally known to have more active disease, with relapses and remissions predominating over slowly progressive symptoms. The course of the disease is similarly more active in the first years, with most patients following the relapsing-remitting pattern of disease progression. Enhancement frequency on MRI has been shown to decrease with increasing age in relapsingremitting MS [45]. In agreement with our findings, a study [34] of 33 patients showed no dependence of alterations to the BRT on the duration of illness.

The effect of therapies used for MS on BRT results cannot be adequately assessed in a study of this design. At one time or another, $80 \%$ of our study participants have received immune-modifying therapy, with most being actively treated with one or the other medication. The only ones not to have received any treatment were the $20 \%$ of patients included in the study with de novo MS diagnosis. As mentioned above, no significant statistical correlation $(p=0.59)$ was found between the two groups.

\section{CONCLUSION}

MRI testing of the brain is undoubtedly the diagnostic modality of choice for diagnosing patients with clinical evidence of MS and sometimes for follow-up. Electrodiagnostic neurophysiological testing is often used for following up patients, as its role in establishing an MS diagnosis has become more limited. The most important finding in this study is that the sensitivity of the BRT is consistent in patients with and without imaged demyelinating lesions in the brainstem. First of all, this suggests that studies of the blink reflex involving heterogeneous groups of diseases (i.e MS, vascular infarctions) should be avoided. The notion of the BRT representing a purely brainstem reflex is again challenged as its long-known, but often forgotten, supratentorial influences resurface. Most other studies have focused on such influences in vascular patients, not MS. However, unlike the case with isolated vascular lesions, the multiplicity in number and the diversity in pathophysiological stage of demyelinating lesions has not allowed any investigator to attempt a more precise correlation of supratentorial lesions with the neurophysiology of the blink reflex. At present, brain MRI may reflect brainstem involvement more appropriately than BRT, regardless of the degree of correlation of each study modality to the clinical picture. Still, newer MRI techniques that are not yet widely used in the community or others yet to be developed may demonstrate what may now be "subimageable" disease in the brainstem of MS patients. Different correlations will then be possible between BRT results, MRI findings and even clinical symptoms or signs. Until then, the need to adequately evaluate the results obtained in the context of each individual patient remains as strong as ever.

\section{ACKNOWLEDGMENTS}

No funding was required for the study. The Blink Reflex Test was performed on patients free of charge. The patients provided their MRI of the brain, which was performed at their own or their insurer's cost as part of their medical care. The manuscript was prepared by the authors themselves without the use of other parties requiring funding.

Frederick Andermann, MD, and John Stewart, MD, contributed in the preparation of the manuscript. Kalliopi Varakis, MD, assisted with issues relating to MRI.

\section{ABBREVIATIONS}

BRT $=$ Blink Reflex Test
BS $=$ Brainstem
$\mathrm{CI}=$ Confidence Interval
MRI $=$ Magnetic Resonance Imaging
MS $=$ Multiple Sclerosis; ms, miliseconds

\section{REFERENCES}

[1] Kimura J. Electrodiagnosis in diseases of nerve and muscle: principles and practice. $3^{\text {rd }}$ ed. New York: Oxford University Press, Inc. 2001; pp. 409-38.

[2] Preston D, Shapiro B. Electromyography and neuromuscular disorders. 1st ed. Butterworth-Heinemann: Boston 1998; pp. 57-62.

[3] Sanders E, Reulen JP, Van der Velde EA, Hogenhuis LA. The diagnosis of multiple sclerosis. Contribution of non-clinical tests. J Neurol Sci 1986; 72(2-3): 273-85.

[4] Cruccu G, Iannetti G, Marx J, et al. Brainstem reflex circuits revisited. Brain 2007; 128: 386-94.

[5] Hopf HC. Topodiagnostic value of brain stem reflexes. Muscle Nerve 1994; 17: 475-84.

[6] Kossev A, Dengler R, Struppler A. Quantitative assessment of the blink reflex in normals. Electromyogr Clin Neurophysiol 1983; 23: 501-11.

[7] Lowitzsch K, Lüder G. Habituation of the blink reflex. Electroencephalogr Clin Neurophysiol 1985; 60: 525-31.

[8] Rossi A, Scarpini C. Gating of trigemino-facial reflex from lowthreshold trigeminal and extratrigeminal cutaneous fibers in humans. J Neurol Neurosurg Psychiatry 1992; 55: 774-80.

[9] Trontelj MA, Trontelj JV. Reflex arc of the first component of the human blink reflex: a single motoneuron study. J Neurol Neurosurg Psychiatry 1978; 41: 538-47.

[10] Hopf HC, Thömke F, Gutmann L. Midbrain vs. pontine medial longitudinal fasciculus lesions: the utilization of masseter and blink reflexes. Muscle Nerve 1991; 14: 326-30.

[11] Marx J, Thoemke F, Fitzek S, et al. Topodiagnostic value of blink reflex R1 changes: A digital postprocessing MRI correlation study. Muscle Nerve 2001; 24: 1327-31.

[12] Kimura J. Alteration of the orbicularis oculi reflex by pontine lesions: study in multiple sclerosis. Arch Neurol 1970; 22: 156-61.

[13] Kimura J. Electrically elicited blink reflex in diagnosis of Multiple Sclerosis - review of 260 patients over a seven year period. Brain 1975; 98(3): 413-26.

[14] Courville J. The nucleus of the facial nerve. The relation between cellular groups and peripheral branches of the nerve. Brain Res 1966; 1: 338-54. 
[15] Fitzek S, Fitzek C, Marx J, et al. Blink reflex R2 changes in lower brainstem - an electrophysiological and MR-imaging study. J Neurol Neurosurg Psychiatry 1999; 67: 630-6.

[16] Ongerboer de Visser BW, Kuypers HGJM. Late blink reflex changes in lateral medullary lesions. Brain 1978; 101: 281-94.

[17] McDonald WI, Compston A, Edan G, et al. Recommended diagnostic criteria for multiple sclerosis: guidelines from the international panel on the diagnosis of multiple sclerosis. Ann Neurol 2001; 50: 121-7.

[18] Simon J, Li D, Traboulsee A, et al. Standardized MR imaging protocol for multiple sclerosis: consortium of MS centers consensus guidelines. Am J Neuroradiol 2006; 27: 455-61.

[19] Deuschl G, Eisen A, Eds. Recommendations for the practice of clinical neurophysiological. IFCN Guidelines. Electroencephalogr Clin Neurophys 1999; Suppl 52: 263-8.

[20] Ferguson IT, Ramsden RT, Lythgoe M. Brain stem auditory evoked potentials and blink reflexes in multiple sclerosis. J Larygol Otol 1985; 99: 677-83.

[21] Kayamori R, Dickins QS, Yamada T, Kimura J. Brainstem auditory evoked potential and blink reflex in multiple sclerosis. Neurology 1984; 34(10): 1318-23.

[22] Khoshbin S, Hallett M. Multimodality evoked potentials and blink reflex in multiple sclerosis. Neurology 1981; 31(2): 138-44.

[23] Kjaer M. The value of brain stem auditory, visual and somatosensory evoked potentials and blink reflexes in the diagnosis of multiple sclerosis. Acta Neurol Scand 1980; 62(4): 220-36.

[24] Lacquaniti F, Benna P, Gilli M, Troni W, Bergamasco B. Brain stem auditory evoked potentials and blink reflexes in quiescent multiple sclerosis. Electroencephalogr Clin Neurophysiol 1979; 47(5): 607-10

[25] Sanders EA, Ongerboer de Visser BW, Barendswaard EC, Arts RJ. Jaw, blink and corneal reflex latencies in multiple sclerosis. J Neurol Neurosurg Psychiatry 1985; 48(12): 1284-9.

[26] Scarpalezos S, Stamboulis E, Eudokimidis J, Siafakas A, Monastiriotis N. Contribution of visual evoked potentials, electronystagmography and the blink reflex to the diagnosis of multiple sclerosis. Rev Neurol (Paris) 1986; 142(10): 753-8.

[27] Simmat G, Gil R, Boissonnot L, Lefèvre JP. The blink reflex and the diagnosis of multiple sclerosis. Clinical correlations in 66 patients. Rev Electroencephalogr Neurophysiol Clin 1985; 15(1): 107-10.

[28] Tackmann W, Ettlin T. Blink reflexes elicited by electrical, acoustic and visual stimuli. II. Their relation to visual-evoked potentials and auditory brain stem evoked potentials in the diagnosis of multiple sclerosis. Eur Neurol 1982; 21(4): 264-9.

[29] Tackmann W, Strenge H, Barth R, Sojka-Raytscheff A. Evaluation of various brain structures in multiple sclerosis with multimodality evoked potentials, blink reflex and nystagmography. J Neurol 1980; 224(1): 33-46.

[30] Lowitzsch K, Kuhnt U, Sackmann C, et al. Visual pattern evoked responses and blink reflexes in assessment of MS diagnosis. J Neurol 1976; 213: 17-32.
[31] Alonso F, Traba A, Roldan R, Esteban A. Brainstem lesion in multiple sclerosis, blink reflex, and brainstem auditory evoked potentials. Arch Neurobiol (Madr) 1992; 55(3): 89-98.

[32] Nazhel B, Irkeç C, Koçer B. The roles of blink reflex and sympathetic skin response in multiple sclerosis diagnosis. Mult Scler 2002; 8(6): 500-4.

[33] Klissourski M, Novachkova S, Tzvetanov P, Alexiev F. Orbicularis oculi reflex abnormalities in patients in patients with multiple sclerosis: a clinical, EMG, and MRI investigation. Electromyogr Clin Neurophysiol 2009; 49(1): 59-63.

[34] Gil R, Lefèvre JP. Electrologic syndromes of alteration of elicited blink reflex in multiple sclerosis. Study of a group of 33 patients (author's transl). Sem Hop 1980; 56(5-6): 214-20.

[35] Fisher MA, Shahani BT, Young RR. Assessing segmental excitability after acute rostra1 lesions: the blink reflex. Neurology 1979 29: 45-50.

[36] Kaplan PE, Kaplan C. Blink reflex: review of methodology and its application to patients with stroke syndrome. Arch Phys Med Rehabil 1980; 61: 30-3.

[37] Kimura J, Lyon LW. Orbicularis oculi reflex in the Wallenberg syndrome: alteration of the late reflex by lesion of the spinal tract and nucleus of the trigeminal nerve. J Neurol Neurosurg Psychiatry 1972; 35: 228-33.

[38] Berardelli A, Accornero N, Cruccu G, Fabiano F, Guerrisi V, Manfredi M. The orbicularis oculi response after hemispheral damage. J Neurol Neurosurg Psychiatry 1983; 46: 837-43.

[39] Dengler R, Kossev A, Gippner C, Struppler A. Quantitative analysis of blink reflexes in patients with hemiplegic disorders. Electroencephalogr Clin Neurophysiol 1982; 53: 513-24.

[40] Kimura J, Wilkinson JT, Damasio H, Adam HR Jr, Shivapour E Yamada T. Blink reflex in patients with hemispheric cerebrovascular accident (CVA). J Neurol Sci 1985; 67: 15-28.

[41] Ongerboer de Visser BW. Comparative study of corneal and blink reflex latencies in patients with segmental or with cerebral lesions. In: Desmedt JE, Ed. Motor Control Mechanisms in Health and Disease. New York: Raven Press 1983: pp. 757-72.

[42] Aramideh M, Ongerboer de Visser BW, Koelman JH, Majoie CB, Holstege G. The late blink reflex response abnormality due to lesion of the lateral tegmental field. Brain 1997; 120: 1685-92.

[43] Meinke U, Ferbert A. Blink reflex in patients with an ischemic lesion of the brain-stem verified by MRI. J Neurol 1993; 241: 37 44 .

[44] Brazis P, Masdeu J, Biller J. Localization in clinical neurology, $3^{\text {rd }}$ ed. Little, Brown and Company (Inc): Boston 1996; vol. 155-250: pp. 449-534.

[45] Filippi M, Wolinsky JS, Sormani MP, et al. Enhancement frequency decreases with increasing age in relapsing-remitting multiple sclerosis. Neurology 2001b; 56: 422-3.

(C) Mikropoulos et al.; Licensee Bentham Open.

This is an open access article licensed under the terms of the Creative Commons Attribution Non-Commercial License (http://creativecommons.org/licenses/by-nc/3.0/) which permits unrestricted, non-commercial use, distribution and reproduction in any medium, provided the work is properly cited. 